\title{
BMC Anesthesiology Reviewer Acknowledgement, 2012
}

Thomas A Rowles

\section{Contributing reviewers}

The editors of BMC Anesthesiology would like to thank all our reviewers who have contributed to the journal in Volume 12 (2012).

\begin{tabular}{lll} 
Fernando Abelha & Dylan Bould & J. Randall Curtis \\
Portugal & Canada & USA \\
Feray Akgül Erdil & Peter Bragge & Giorgio Danelli \\
Turkey & Australia & Italy \\
Roberto Arcioni & Stefan Breitenstein & Alberto De Armendi \\
Italy & Switzerland & USA \\
Angela Bader & Serge Brimioulle & Daniel De Backer \\
USA & Belgium & Belgium \\
Alberto Barbieri & Peter Brindley & Frederik De Buck \\
Italy & Canada & Belgium \\
Georgia Beasley & Ethan Bryson & Germano De Cosmo \\
USA & USA & Italy \\
Dan Benhamou & Mehmet Fatih Can & Phillip Dellinger \\
France & Turkey & USA \\
Horst Bickel & Somrat Charuluxananan & Paul D'Orazio \\
Germany & Thailand & USA \\
Tom Birkenhäger & Christian Fynbo Christiansen & Volker Dörges \\
Netherlands & Denmark & Germany \\
Simon Body & Eric Chudler & Martin Dworschak \\
USA & USA & Austria \\
Ben Boedeker & Francois Clergue & Sam Eldabe \\
USA & Switzerland & United Kingdom \\
Francis Bonnet & Mark Coburn & Thomas Engelhardt \\
France & Germany & United Kingdom \\
Judith Bosmans & Richard Cooper & Lorella Fanti \\
Netherlands & Canada & Italy \\
\hline Correspondence: Tom.Rowles@biomedcentral.com & & \\
BioMed Central, Floor 6, 236 Gray's Inn Road, London & WC1X8HB, United & \\
Kingdom & & \\
\hline & & \\
\hline & &
\end{tabular}


Emanuele Ferrero

Italy

John Fiadjoe

USA

Paul Firth

USA

Dominique Fletcher

France

Elisabeth Gaertner

France

Luis Gaitini

Israel

Ronald George

Canada

Timothy Girard

USA

Allan Gottschalk
USA

Bruce Green

Australia

Burak Guclu

Turkey

Freedom Nkhululeko Gumedze

South Africa

Ashraf Habib

USA

Robert G Hahn

Sweden

Kaoru Hara

Japan

James Heavner

USA

David Herd

Australia

Jochen Hinkelbein

Germany

Luc Hondeghem

Belgium

Yu-Hsi Hsieh

Taiwan

Luiz Eduardo Imbelloni

Brazil

Fabian Jaimes

Colombia
Jerzy Jankun

USA

Bernd Jilma

Austria

Ken Johnson

USA

Hiroyuki Kinoshita

Japan

Naohiro Kurotaki

Japan

Giovanni Landoni

Italy

Tae Hoon Lee

South Korea

Hendrikus Lemmens

USA

Alyn Lewis

United Kingdom

Vladimir Lomivorotov

Russian Federation

Rebekah Lucas

USA

Marco Luchetti

Italy

Zhengliang Ma

China

Flavia Machado

Brazil

Lynne Maxwell

USA

Colin Mccartney

Canada

William Mcgee

USA

Darryl Mcgill

Australia

Graeme Mcleod

United Kingdom

Vincent Minville

France

Sukanya Mitra

India

Jeffrey Mogil

Canada
Iain Moppett

United Kingdom

Hany Mowafi

Saudi Arabia

Eveline Nueesch

United Kingdom

Doris Østergaard

Denmark

Owen O'Sullivan

Ireland

Stephen Pastores

USA

Rupert Pearse

United Kingdom

Anders Perner

Denmark

Ville Pettilä

Finland

Philip Peyton

Australia

Hemanshu Prabhakar

India

Douglas Raines

USA

Ramachandran Ramani

USA

Saifudin Rashiq

Canada

Jochen Renner

Germany

Daniel Reuter

Germany

Emanuel Rivers

USA

Edgar Alfonso Romero-Sandoval USA

Meg Rosenblatt

USA

Leif Saager

USA

Sanna Salanterä

Finland

Michael Sander

Germany 
Volkher Scharnhorst

Netherlands

Stefan Schraag

United Kingdom

Frederique Servin

France

Rajeev Sharma

India

Tatjana Simurina

Croatia

Frank Skorpen

Norway

Peter Smitham

United Kingdom

Luzius A Steiner

Switzerland

Konstantinos Stroumpoulis

Greece

Bala Subramaniam

USA
Anna Taddio

Canada

Yoshihiro Takasugi

Japan

Mark Tommerdahl

USA

Angela Truong

USA

David Turnbull

United Kingdom

Todd Vanderah

USA

Luigi Vetrugno

Italy

Juan Viñoles

Spain

Thomas Volk

Germany

Christian Von Heymann

Germany
Deborah Wagner

USA

Zen'Ichiro Wajima

Japan

Thomas Weber

Austria

Manuel Wenk

Germany

Chris Winkelman

USA

Sandhya Yaddanapudi

India

Wei-Hsian Yin

Taiwan

doi:10.1186/1471-2253-13-3

Cite this article as: Rowles: BMC

Anesthesiology Reviewer Acknowledgement, 2012. BMC Anesthesiology 2013 13:3. 